NASA/TM-1998-208488

\title{
A Simplified Model for the Investigation of Acoustically Driven Combustion Instabilities
}

D. Dane Quinn

The University of Akron, Akron, Ohio

Daniel E. Paxson

Lewis Research Center, Cleveland, Ohio

Prepared for the

34th Joint Propulsion Conference

cosponsored by AIAA, ASME, SAE, and ASEE

Cleveland, Ohio, July 12-15, 1998

National Aeronautics and

Space Administration

Lewis Research Center 
Available from

NASA Center for Aerospace Information 7121 Standard Drive

Hanover, MD 21076

Price Code: A03
National Technical Information Service 5287 Port Royal Road Springfield, VA 22100 
AIAA-98-3764

\section{A SIMPLIFIED MODEL FOR THE INVESTIGATION OF ACOUSTICALLY DRIVEN COMBUSTION INSTABILITIES}

\author{
D. Dane Quinn * \\ Dept. of Mechanical Engineering \\ The University of Akron \\ Akron, $\mathrm{OH}$ 44325-3903
}

\author{
Daniel E. Paxson $t$ \\ NASA Lewis Research Center \\ MS 77-1 \\ Cleveland, $\mathrm{OH} 44135$
}

\begin{abstract}
A simplified one-dimensional model of reactive flow is presented which captures features of aeropropulsion systems, including acoustically driven combustion instabilities. Although the resulting partial differential equations are one-dimensional, they qualitatively describe observed phenomena, including resonant frequencies and the admission of both steady and unsteady behavior. A number of simulations are shown which exhibit both steady and unsteady behavior, including flame migration and thermo-acoustic instabilities. Finally, we present examples of unsteady flow resulting from fuel modulation.
\end{abstract}

\footnotetext{
*Summer Faculty Fellow at NASA Lewis Research Center quinn@uakron.edu

†Member AIAA, dpaxson@lerc.nasa.gov
}

\section{NOMENCLATURE}

$$
\begin{array}{rlrl}
\text { Sc } & =\frac{k}{D} & \frac{\text { viscosity }}{\text { mass diffusion coefficient }}, \\
\text { Le } & =\frac{\left(\lambda / c_{p}\right)}{D} & \frac{\text { thermal diffusion coefficient }}{\text { mass diffusion coefficient }}, \\
\beta & =\frac{\left(Q / c_{p}\right) y_{0}}{T_{0}} & \text { heat-release, } \\
\theta & =\frac{E}{R T_{0}} & \text { activation energy, } \\
\mu & =\frac{u_{0}}{c_{0}}=\frac{t_{a}}{t_{0}} & \frac{\text { acoustic time scale }}{\text { characteristic time scale }} \\
\delta & =\frac{D}{\rho_{0} x_{0} u_{0}}=\frac{t_{0}}{t_{d}} & \frac{\text { characteristic time scale }}{\text { diffusive time scale }} \\
\Delta=\frac{K x_{0}}{u_{0} e^{\theta}}=\frac{t_{0}}{t_{r}} & \frac{\text { characteristic time scale }}{\text { reactive time scale }} \\
\gamma=\frac{c_{p}}{c_{v}} & \text { specific-heat ratio, }
\end{array}
$$

\section{INTRODUCTION}

The current drive toward lean operating, low-emission, low-loss combustors in next generation aeropropulsion systems makes them susceptible to various instabilities and makes the understanding of those instabilities critical. Combustion instabilities, specifically acoustically driven instabilities, often arise from the interaction between the energy released by the chemical reactions and the underlying fluid flow. This coupling can dramatically alter the dynamical behavior of the combustion process, and result in unstable behavior. Typical combustion instabilities in propulsion systems can often be characterized by oscillatory pressure fluctuations coupled with an unsteady heat release. This instability can lead to flame blowout, reduced performance, and decreased engine life. 
Although the magnitude of the pressure oscillations can be substantial, the total energy associated with the unsteady behavior is typically only a small fraction of the total energy generated by the combustion process. As a result, the pressure fluctuations can often be identified with the acoustic modes of the enclosed volume. The acoustic behavior of the propulsion system induces an oscillatory release of heat, which in turn drives the acoustic modes, or vice versa.

The fundamental equations of reactive gas dynamics can be described as a set of reaction-diffusion equations governing the energy release associated with the combustion and transport of the chemical species, coupled to the Navier-Stokes equations for the underlying fluid flow, subject to an equation of state. Because of the complexity of both the fluid and transport equations, as well as the coupling mechanisms, the ability to model and accurately predict the dynamical behavior and performance of propulsion systems is often limited. As a result, the simulation and characterization of unstable combustion behavior is critical to the development of future propulsion systems and is the subject of intense interest [1].

A common approach to modeling thermo-acoustic combustion instabilities is to simplify the governing equations to those of one-dimensional flow $[1,2]$. The use of a constitutive model with a sensitive time-lag formulation is then employed to couple the unsteady heat release with the underlying fluid flow [3]. As a result, the time-lag, or $n-\tau$ formulation, eliminates the transport equations which govern the chemical kinetics, and reduces the complexity of the resulting model. Although this reduction presents a simplification of the model, it has met with great success in characterizing these instabilities. Despite this success, we study these instabilities with the inclusion of simplified chemical kinetics. Our goal is to obtain fundamental insight into the behavior of these systems, in particular the coupling between the unsteady heat release and the acoustic response.

This work presents a numerical investigation of both the convection and transport equations governing one-dimensional reactive flow. These equations represent a simplified model for the flow in aeropropulsion combustors, and hence cannot capture such phenomena as flow oscillations driven by shear layer instabilities or the liquid-fuel vaporization process. However, they nonetheless exhibit self-excited thermo-acoustic instabilities and can provide a tool for studying the qualitative behavior of such systems, including the sensitivity of the system to parametric variations in the convection and transport parameters. In particular, we study the dynamics of this system with an Arrhenius model for the chemical kinetics and a description of the flame holder. The simulation will be described, including the governing equations, justification for the simplifications, and integration techniques. The results will be presented and contrasted with respect to the development of instabilities.

\section{GOVERNING EQUATIONS}

We consider a premixed gas flowing in a finite length combustor with constant cross sectional area, which undergoes a single-step irreversible chemical reaction. In this formulation, we assume that the mixture contains a single deficient component, the amount of which controls the reaction. In addition, this reactive component is assumed to exist in an inert bath, in which the total concentration of the reactive component is small-as the combustion process occurs, the overall gas parameters of the mixture remain unchanged.

In a single spatial dimension, the basic nondimensional equations of motion governing reactive gas dynamics are:

$$
U_{t}+F_{x}=S
$$

with:

$$
\begin{aligned}
& U=\left[\begin{array}{c}
\rho \\
\rho u \\
\rho E \\
\rho y
\end{array}\right], \quad F=\left[\begin{array}{c}
\rho u^{2}+\frac{p}{\gamma \mu^{2}} \\
\rho u E+\frac{(\gamma-1)}{\gamma} p u \\
\rho u y
\end{array}\right], \\
& S=\left[\begin{array}{c}
0 \\
\dot{Q}+\operatorname{Le}\left(\delta T_{x}\right)_{x}+\frac{4}{3}(\gamma-1) \mu^{2} \operatorname{Sc} \delta u_{x}^{2} \\
-\dot{m}+\left(\delta y_{x}\right)_{x}
\end{array}\right] .
\end{aligned}
$$

$E$ represents the total energy and is defined as:

$$
E=\frac{T}{\gamma}+\mu^{2} \frac{(\gamma-1)}{2} u^{2}
$$

Time is nor dimensionalized by the acoustic time scale, so that $\mu=1.00$, and $z_{(\cdot)}=\partial z / \partial(\cdot)$. These equations are completed by the ideal gas law, $p=\rho T$.

We ascume that there is no external heat transfer, although these equations will be augmented below to include the effect of a flame holder. These equations are written in conservation form, with $\rho(x, t)$ and $u(x, t)$ the total density and mass weighted velocity, respectively, of the gas. The temperature is described by $T(x, t), p(x, t)$ is the pressire, and $y(x, t)$ represents the mass fraction of the react int in the combustible gas.

The sc urce terms within $S$ contain the thermodynamic effec $s$ of the reactive flow, as well contributions from diffusional terms. $\dot{Q}$ represents the rate of heat release by he chemical reaction, while $\dot{m}$ is the mass conversion ate of the unreacted fuel. These equations have been used by a number of authors to study flame propagation through high activation energy asymptotics $[4,5,6,7,3]$ and the behavior of premixed combustion in wave rot irs [9].

The rondimensionalization has been performed with respect to the unreacted gas and the dimensional combustor length $l$. At the inlet, $x=0$, we impose $y(0, t)=1$. The boundary conditions on the pressure and temper ature are dependent on the geometry of the combustion chamber. In addition, physically we expect

2

American Institute of Aeronautics and Astronautics 
$y(x, t) \rightarrow 0$ as $x \rightarrow l$. It should be noted that the diffusive time scale is not necessarily controlled by the fluid properties but may well be controlled by the flowfield (e.g. through turbulent diffusion).

\section{Heat-release}

We describe the instantaneous rate of heat release, $\dot{Q}$, associated with the combustion process as a one-step, irreversible reaction, where $\dot{Q}$ is proportional to the mass conversion rate of the unreacted fuel. In addition, we assume that $\dot{m}$ is proportional to the mass of unreacted fuel, with a temperature dependent reaction rate:

$$
\begin{aligned}
\dot{Q} & =\beta \dot{m}, \\
& =\beta((\Delta \rho y) \cdot k(T)),
\end{aligned}
$$

where $\beta$ is the nondimensional heat release parameter and $\dot{m}$ is the mass conversion rate of the unreacted fuel. $\rho$ and $y$ are the density of the gas and the mass fraction of the premixed fuel, respectively, so that the quantity $\rho y$ physically represents the mass per unit volume of fuel available to the combustion process. $\Delta$ is a parameter associated with the rate of mass conversion and $k(T)$ is the temperature-dependent component of the reaction rate.

Mixture thermodynamics. The thermodynamic properties of the premixed gas are contained within the parameters $\beta$ and $\Delta$. If we subsequently consider varying mixtures of oxidant and reactant, both parameters are limited above by some maximum values, say $\beta_{\max }$ and $\Delta_{\max }$.

The flame temperature resulting from the combustion process scales with $\beta$, the nondimensional heat release, so that the temperature at the exit is approximately $T_{1}=T(1, t)=T_{0}+\beta$, where $T_{0}=T(0, t)$. As such, for a given reactant, the maximum value of $\beta$ roughly corresponds to stoichiometric conditions, so as $\beta$ approaches $\beta_{\max }$, the equivalence ratio of the mixture approaches unity. Also, $\Delta$, the Damköhler number, scales the rate of the chemical reaction. These quantities parallel the equivalence ratio and the heat content of the premixed fuel.

Chemical kinetics. In this simulation we model the chemical reaction by a single-step, first-order Arrhenius model, so that the temperature dependent reaction rate is:

$$
k(T)_{\text {Arrhenius }}=e^{\theta(1-1 / T)},
$$

with $\theta$ the activation energy [4]. In addition, we also incorporate an ignition temperature $T_{i}$, below which no combustion occurs. As a result, $k(T)$ takes the form:

$$
k(T)= \begin{cases}0, & T<T_{i} \\ e^{\theta(1-1 / T)}, & T \geq T_{i}\end{cases}
$$

With this choice for $k(T)$, the ratio between the maximum rate of reaction at $T=T_{1}$ and the initial rate of reaction at $T=T_{i}$ is approximately:

$$
\frac{k\left(T_{1}\right)}{k\left(T_{i}\right)} \sim \exp \left\{\theta\left(\frac{1}{T_{i}}-\frac{1}{T_{0}+\beta}\right)\right\} .
$$

\section{Flame Holder}

In the absence of a flame holder, a stable flame requires a delicate balance between the upstream diffusion of the flame and the downstream convection of the fluid. As a result, for most all gas parameters, this balance will not occur and the flame will either propagate upstream to the inlet (flashback), or propagate downstream and out of the combustor, a condition known as blow-out. The flame holder acts to limit the propagation of the flame upstream. We note that flow effects, such as a spatially varying flow velocity due to cross-sectional area variations, can also serve to anchor the flame.

We assume that the effects of the flame holder are twofold: (i) the diffusion constant $\delta$ becomes spatially dependent, resulting from increased turbulent diffusivity downstream of the flame holder, and (ii) the flame holder transfers heat to and from the fluid. We incorporate both effects in this model.

Diffusional effects. We assume that the flame holder acts to increase the diffusion due to induced turbulent mixing. This implies that the scale of the turbulent eddies is larger than the width of the laminar flame zone. As a result, we model the spatial distribution of $\delta(x)$ as:

$$
\delta(x)=\delta_{1}\left[\frac{1}{2}\left(1-\frac{\delta_{0}}{\delta_{1}}\right)(\tanh (20(x-a))+1)+\frac{\delta_{0}}{\delta_{1}}\right] .
$$

In the limits $x \rightarrow \pm \infty$ the diffusional coefficient approaches $\delta_{0}$ and $\delta_{1}$ respectively.

Thermodynamic effects. The heat transfer effects are assumed to be spatially dependent as well, of the form:

$$
\begin{aligned}
f_{\text {thermal }}(T(x, t), x)= & g(x)\left(T_{f}-T(x, t)\right) \\
= & f_{0} \exp \left[\left(1-(50(x-a))^{4}\right)\right] \times \\
& \left(T_{f}-T(x, t)\right)
\end{aligned}
$$

where $g(x)$ is the local convection transfer coefficient, and $T_{f}$ is the nominal temperature for the flame holder [10]. Thus $g(x)$ describes the spatial distribution of the convective effects of the flame holder. We augment the energy equation, by $f(T, x)$. In addition to the increased diffusional effects discussed above, the flame holder acts to limit the propagation of thermal energy upstream of the flame. This form for the heat transfer coefficient provides an analytic approximation which is localized in the neighborhood of $x=a$. 


\section{NUMERICAL SIMULATIONS}

With prescribed initial conditions, the system governing reactive gas dynamics in the form of Eq. (1) is simulated using MacCormack's method [11], which is secondorder accurate in both space and time. The flow is solved in the interval $0 \leq x \leq 1$ for $t \geq 0$, subject to characteristic-based boundary conditions [12], and the diffusional terms are incorporated as a source using a second order spatial differencing scheme. To verify the qualitative accuracy of our integration scheme we have varied both the number of grid points, as well as the size of the time step.

Unless noted, we assume $\gamma=1.40$ and choose the flow parameters:

$$
\begin{aligned}
& \mathrm{Sc}=1.00, \quad \text { Le }=1.00, \quad \mu=1.00, \\
& \beta=1.50, \quad \theta=2.00, \quad T_{i}=1.00 \text {. }
\end{aligned}
$$

The flame holder located to be at $25 \%$ of the combustor length $(a=0.25)$, with:

$f_{0}=0.1, \quad T_{f}=1.00, \quad \delta_{1}=0.01, \quad \frac{\delta_{0}}{\delta_{1}}=0.025$.

In the following, we present several simulations to illustrate the dynamical characteristics of the model, including both steady and unsteady behavior. In addition, we consider both choked and open inlet conditions, as well as a number of different parametric conditions. In the figures, darker shades indicate lower amplitudes.

\section{Choked-open Boundary Conditions}

The inlet conditions are choked and isothermal, so that:

$$
\rho(0, t) u(0, t)=0.20, \quad T(0, t)=0.75,
$$

while the exit is open:

$$
p(1, t)=1.00 \text {. }
$$

The remaining boundary conditions are determined from the interior of the domain of integration $x \in[0,1]$. In Figure 1, we show the steady response for $\Delta=2.50$. We note that this response is stable.

\section{Flame Migration}

By starting the flame away from its equilibrium position, we can also observe the transient behavior of the flame. As seen in Figure 2, combustion initially occurs at $x=0.40$, well away from the flame holder located at $x=0.20$. The simulation parameters are chosen as above, with $\Delta=1.50$. As time evolves, the flame moves upstream, eventually stabilizing on the flame holder.

\section{Open-open Boundary Conditions}

We now apply open boundary conditions and assume the flow is isothermal at the inlet, so that at $x=0$ :

$$
p(0, t)=p_{0}=1.05, \quad T(0, t)=0.90,
$$

while the exit is open:

$$
p(1, t)=1.00,
$$

which implias a $5.0 \%$ pressure drop across the combustor length. The remaining boundary conditions are determined from the interior of the domain of integration $x \in[0,1]$.

\section{Unsteady Response}

For $\Delta=1.50$, the asymptotic solution is unsteady, and the fundamental mode is excited (see Figure 3). In Figure $3 \mathrm{a}$ we show the time trace of the pressure at $x=0.25$. As time increases the amplitude of the pressure oscillations initially grow before limiting around $t=35$. For comparison with Figure 5, in Figures $3 c$,d we show the steady solution, which for this value of $\Delta$ is unstable.

In Figure 4 we show the time-varying components of the flow, denoted by $(\cdot)^{\prime}$, including the unsteady rate of heat release $\dot{Q}^{\prime}(x, t)$. We define the local Rayleigh index $\mathcal{R}(x, t)=\dot{Q}^{\prime}(x, t) \cdot p^{\prime}(x, t)$, and further define the Rayleigh index as the time average of this local index over one oscillation:

$$
G(x) \equiv \frac{1}{T} \int_{T} \mathcal{R}(x, t) d t,
$$

where $T$ is the period of oscillation. $\mathcal{R}(x, t)$ represents the work done to the fluid by the chemical heat release at time $t$ and spatial location $x$. As time evolves the local Rayleigh index, and hence the work done,takes on both positive and negative values. However, $G(x)$ represents the average work done over one cycle. The well-known Rayleigh's criterion states that if $G(x)>0$ energy is added to the system and the amplitude of the pressure oscillation is expected to increase, while for $G(x)<0$ the flame-acoustic interaction removes energy from the system. As expected, examination of Figure 4 indicates that over most of the flow field the unsteady heat release is in-phase with the oscillating pressure component-the Rayleigh index is positive and we indeed find an unsteady response.

\section{Parametric Variations}

Darling, et al. experimentally studied the acoustic response in several combustor configurations [13]. In their work, it was noted that " ... the resonances were very sensitive to shanges in the mean flow conditions, and the effects of these changes were not al ways consistent." For example, at low inlet temperatures the amplitude of a resonant acoustic mode was increased as the equivalence ratio was increased. However, for larger inlet temperatures the rex onant peak was decreased as the equivalence ratio was in rreased.

Although, as mentioned above, we do not attempt to make dir ect comparisons between our fuel parameters $(\beta, \Delta)$ ind the equivalence ratio and heat capacity, we do note that as we change $\beta$ and $\Delta$ this model possesses similar qualitative behavior. For both $\Delta=\mathbf{0 . 7 5}$ and $\Delta=4.00$ the asymptotic response, shown in Figure 5 , is stiady, while, as seen in Figures 3 and 4 , an unsteady response exists for $\Delta=1.50$. This type of behavior is common-there is an intermediate range of $\Delta$ for whicl the steady-state solution is unstable. For 


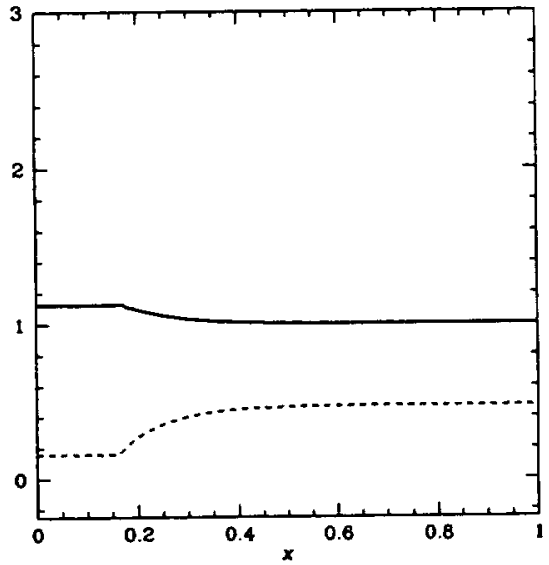

a) $\left(p_{\text {eq }}(x), u_{e q}(x)\right)$ vs. $x$

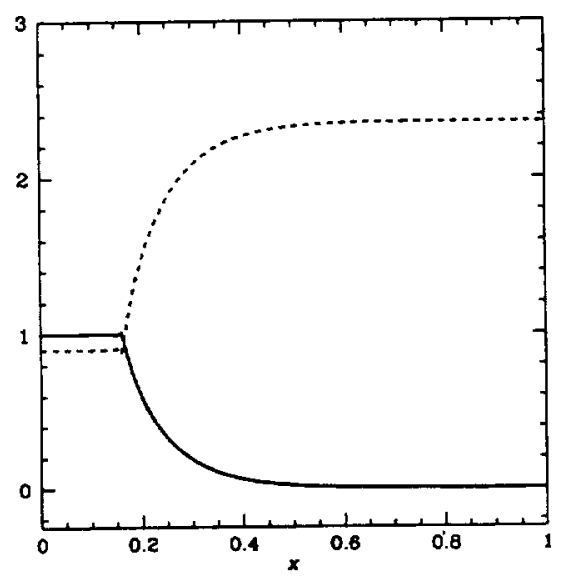

b) $\left(T_{e q}(x), y_{e q}(x)\right)$ vs. $x$

FIGURE 1: Choked-open boundary conditions - steady response $(\Delta=2.50)$. The flow velocity and temperature are shown dashed in a) and b) respectively, while the pressure and species fraction are solid curves.

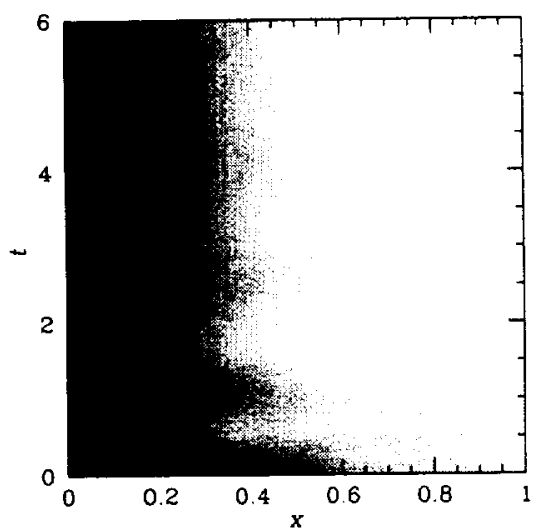

a)

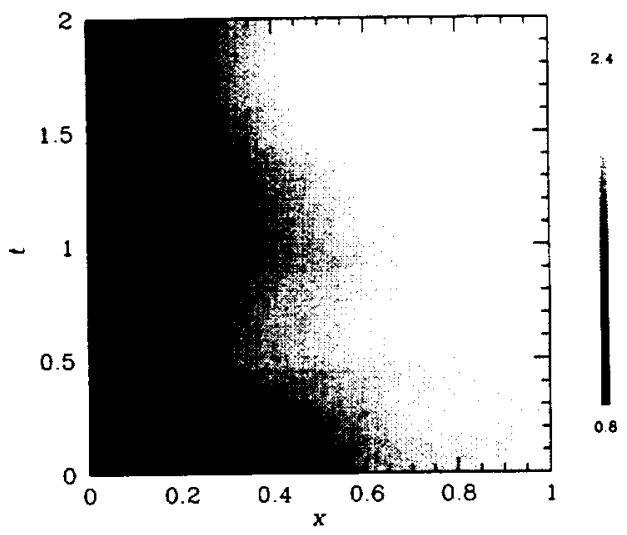

b)

FIGURE 2: Flame migration and stabilization with choked-open boundary conditions. Figure b) is an expanded view for $0 \leq t<2$ which emphasizes the migration of the flame, initially located at $x=0.4$, upstream to the flame holder at $x=0.2$. 


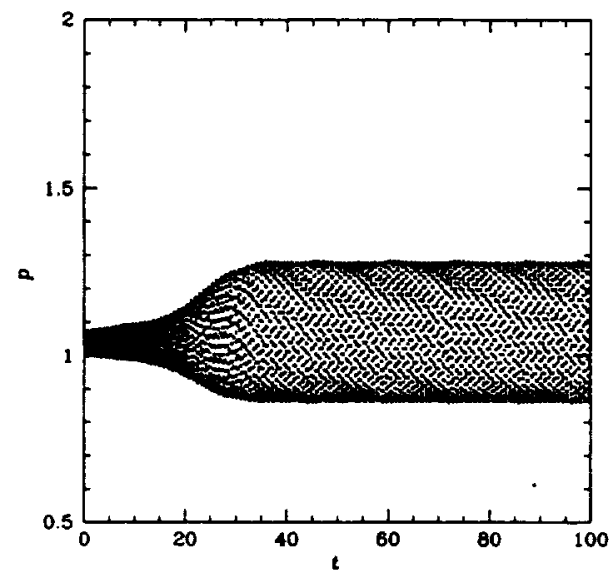

a) $p(0.25, t)$ vs. $t$

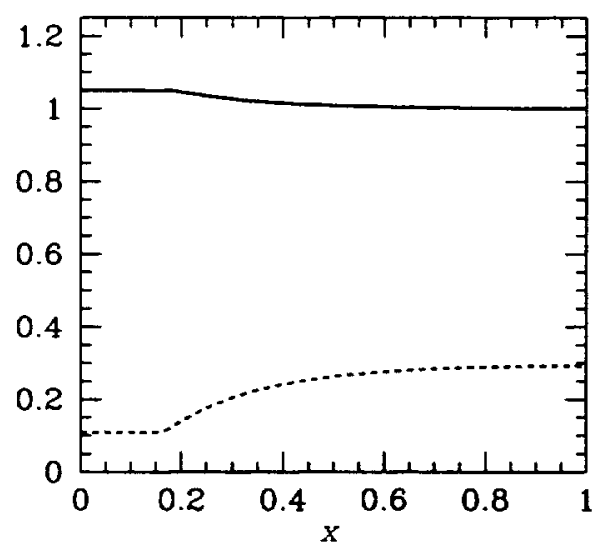

c) $\left(p_{e q}(x), u_{e q}(x)\right)$ vs. $x$

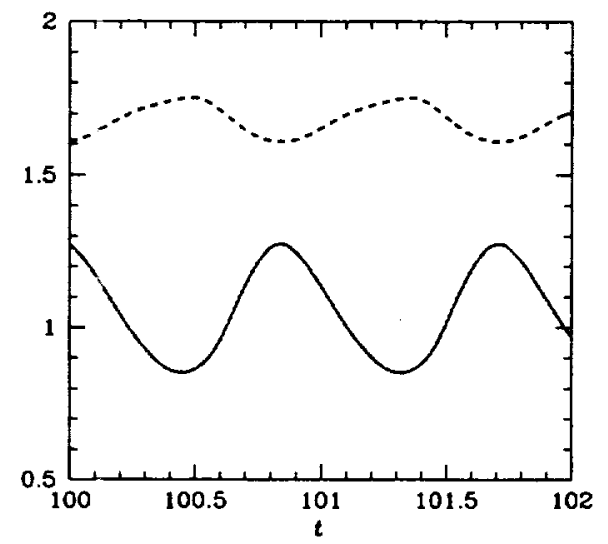

b) $(p(0.25, t), T(0.25, t))$ vs. $t$

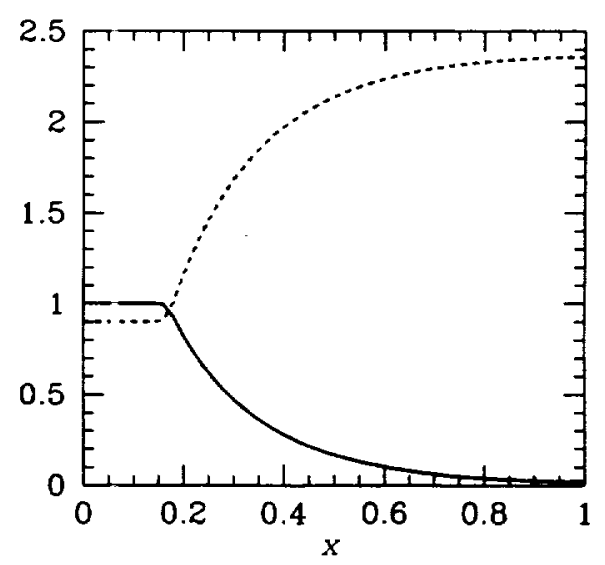

d) $\left(T_{e q}(x), y_{e q}(x)\right)$ vs. $x$

FIGURE 3: Open-open boundary conditions $\left(\delta_{1}=0.02\right)$. For $\Delta=1.25$ the response is unsteady. Figure a) presents the growth of the pressure response as time increases, eventually approaching a periodic oscillation. In b) the steadystate oscillation in pressure and temperature at $x=0.25$ are shown as the solid and dotted line respectively, while in c) and d) we show the time-independent profile which is unstable. The flow velocity and temperature are shown dashed in c) and d) respectively, while the pressure and species fraction wre solid curves. 


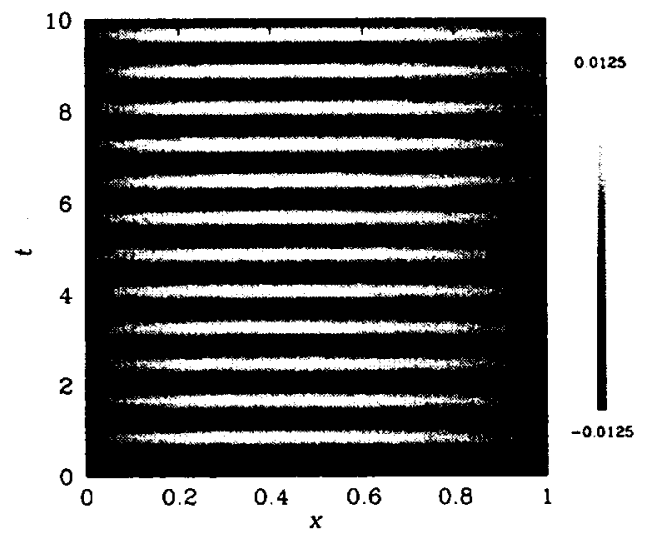

a) $p^{\prime}(x, t)$

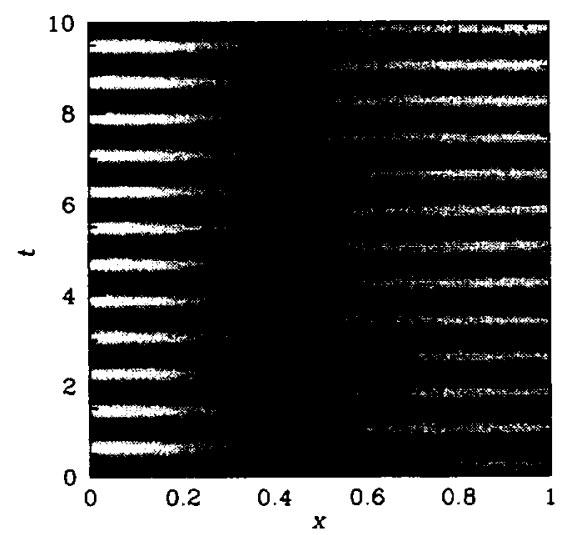

c) $u^{\prime}(x, t)$

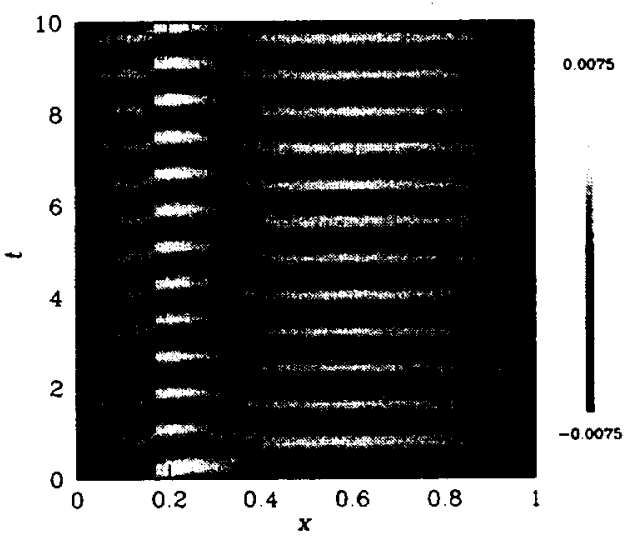

b) $T^{\prime}(x, t)$

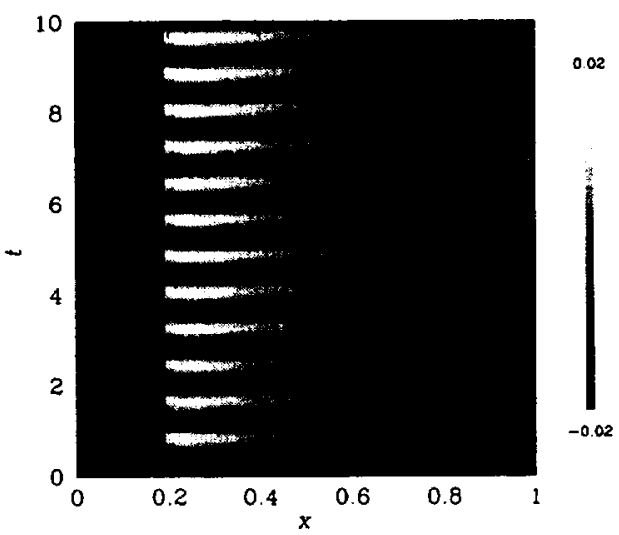

d) $\dot{Q}^{\prime}(x, t)$

FIGURE 4: Open-open boundary conditions $\left(\delta_{1}=0.02\right)$. Unsteady components of the response shown in Figure 3. The time interval shown is while the amplitudes of the oscillations are small. As seen previously in Figure 3 , large amplitude oscillations eventually develop. 
$\Delta$ sufficiently small or large, the steady-state is stable. Reflecting back on our discussion between the relationship between $\beta$ and $\Delta$, and the equivalence ratio, this implies that for near-stoichiometric mixtures, our model leads to an unsteady asymptotic response. As $\Delta \rightarrow 0$ we numerically encounter flame blowout, while large $\Delta$ admits the possibility of flashback.

Finally, we note that for $\theta=2.0$ the ratio between the final and initial burning rate is approximately 3.2 . If we decrease the activation energy to $\theta=1.0$, so that ratio between the final and initial burning rate is approximately 1.8 the response is stable for all values of $\Delta$.

\section{Fuel Flow Modulation}

Looking forward to possible attempts to implement control strategies based on fuel modulation, we study the effects of small amplitude oscillations in the fuel species parameter $y$ at the inlet:

$$
y(0, t)=1.0+0.01 \sin (2 \pi \omega t)
$$

In Figure 6a, we show the amplitude of the pressure oscillation at $x=0.25$ as the modulation frequency $\omega$ varies from $\omega=0.25$ to $\omega=1.00$. When the fuel species is modulated near the fundamental acoustic frequency at $\omega \sim 0.6$, modulation amplitudes of $1 \%$ lead to large amplitude acoustic oscillations. Figure $6 \mathrm{~b}$ presents the growth of the pressure oscillation for $\omega=0.6$. We note that in these simulations the flame zone is located at approximately $20 \%$ of the combustor length. As a result, diffusional effects reduce the amplitude of the fuel modulation at the flame zone when compared to the inlet fluctuations. Thus we expect that if the fuel was modulated slightly upstream of the flame zone, rather than at the inlet, the resulting acoustic response would increase further.

\section{SUMMARY}

This work focuses on numerical simulations of reactive flow which, while retaining the simplification of onedimensional flow, contains equations for species transport as well as fluid convection and diffusion. As evidenced by the representative figures shown, it is possible to study thermo-acoustic instabilities, flame evolution, and the behavior resulting from parametric variations. In addition, the model for the chemical kinetics contains two effects-Arrhenius temperature dependence and an extinction temperature. We also investigate the dynamical behavior with the inclusion of both thermodynamic and diffusional effects from the flame holder.

Examination of the unsteady temperature distribution indicates two distinct regions along the width of the combustor-i) the flame zone, in which large temperature variations occur due to the chemical reactions, and ii) remainder of the combustor, in which the temperature variations are, in fact, almost isentropic.

Although the model is too simplified to obtain quantitative comparisons with the phenomena observed in experimental tests, it provides an ideal framework to study the qualitative behavior of thermo-acoustic coupling and caft be used as a basis for the investigation of the control of these oscillations and the implementation of various control strategies, including modulation of the fuel flow.

\section{REFERENCES}

[1] Oyediran, A., Darling, D., and RadhakrISHNAN, $K$. Review of combustion-acoustic instabilities. Technical Memorandum NASA TM107020, AIAA-95-2469, NASA, August 1995. 31st AIAA/ASME/SAE/ASEE Joint Propulsion Conference.

[2] Mcmanus, K. R., Poinsat, T., and Candel, S. M. A review of active control of combustion instabilities. Progress in Energy and Combustion Science, 19:1-29, 1993.

[3] Crocco, L. and Cheng, S.-I. Theory of Combustion Instability in Liquid Propellant Rocket Motors. Butterworths Scientific Publications, London, 1956.

[4] Buckmaster, J. D. And Ludford, G. S. S. Theory of Laminar Flames. Cambridge University Press, Cambridge, 1982.

[5] Clavin, P., Pelcé, P., and He, L. Onedimensional vibratory instability of planar flames propagating in tubes. Journal of Fluid Mechanics, 216:299-322, 1990.

[6] Ledder, G. AND KapilA, A. K. The response of premixed flames to pressure perturbations. Combustion Science and Technology, 76:21-44, 1991.

(7) Matalon, M. and Matkowsky, B. J. Flames as gasdynamic discontinuities. Journal of Fluid $\mathrm{Me}$ chanics, 124:239-259, 1982.

[8] McINT)SH, A. C. The effect of upstream acoustic forcing and feedback on the stability and resonance behavicr of anchored flames. Combustion Science and Teshnology, 49:143-167, 1986.

[9] Nalim, M. R. AND Paxson, D. E. A numerical investigation of premixed combustion in wave rotors. ASME Journal of Engineering for Gas Turbines and Power, 119(3):668-675, 1997.

[10] Entez. aM, B., Van Moorhem, W. K., and MaJDALAN I, J. Modeling of a Rijke-tube pulse combustor using computational fluid dynamics. AIAA Paper AIAA 97-2718, AIAA, July 1997. 33rd AIAA/ASME/SAE/ASEE Joint Propulsion Conference.

[11] Anderson, D. A., Tannehill, J. C., AND PletCien, R. H. Computational Fluid Mechanics and Heat Transfer. McGraw-Hill, New York, 1984. 


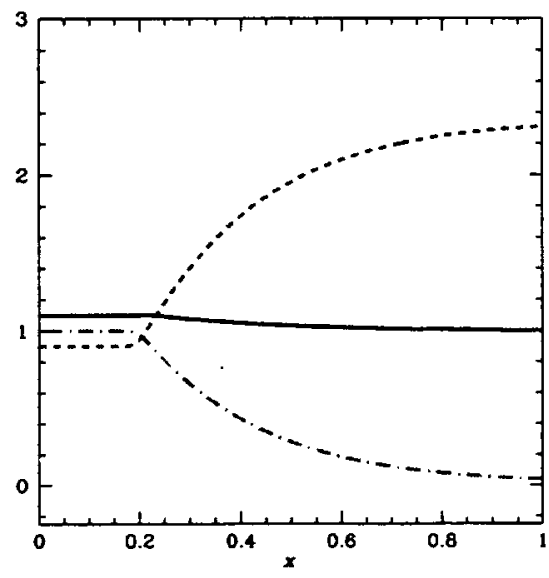

a) $\Delta=0.75$

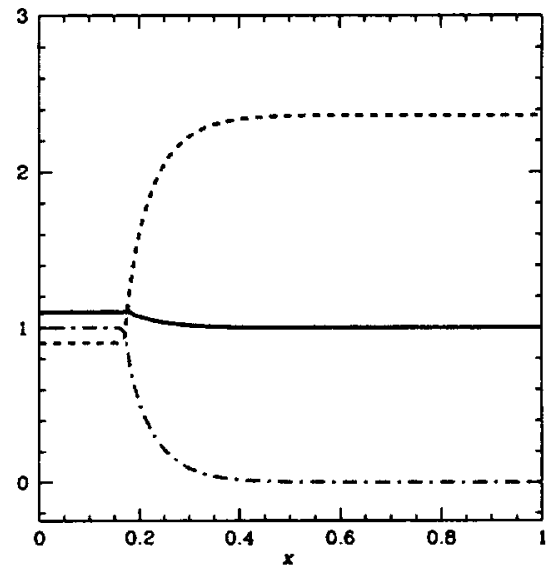

b) $\Delta=4.00$

FIGURE 5: Open-open boundary conditions-for $\Delta=0.75$ and $\Delta=4.00$ the time-independent response is stable, shown in a) and b) respectively. As $\Delta$ increases the length of the flame zone decreases. In each figure the states are denoted by: pressure ___ temperature .... . ; velocity _ _ _; species _...

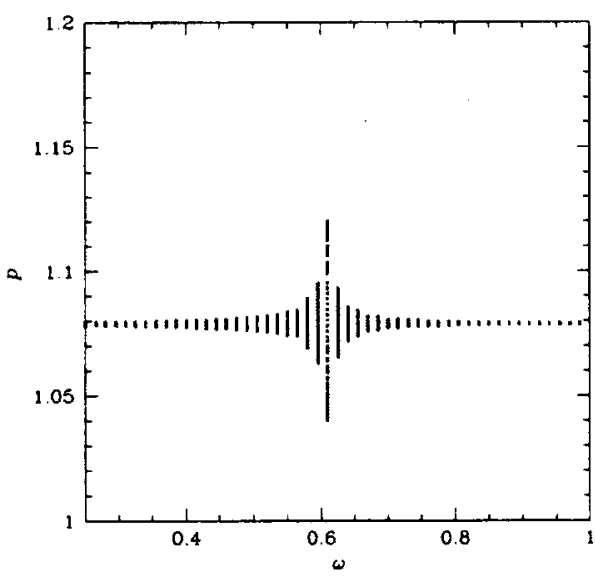

a) $y(0, t)=1.0+0.01 \sin (2 \pi \omega t)$

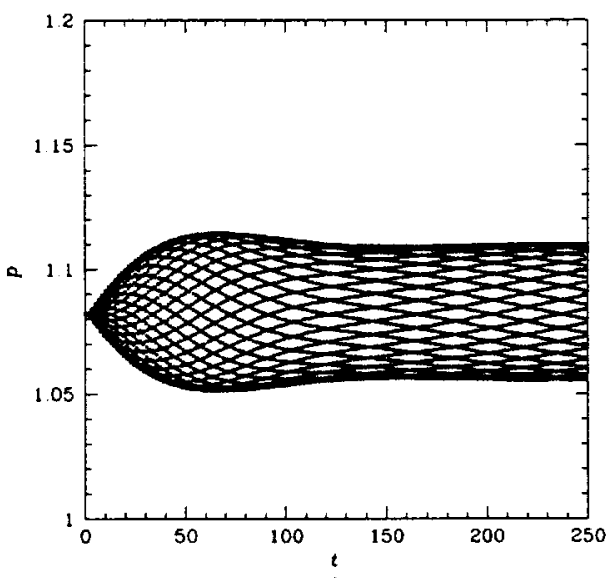

b) $p(0.25, t) ; \omega=0.60$

FIGURE 6: Acoustic oscillations resulting from fuel flow modulation $(\theta=1.50, x=0.25)$. In a) the amplitude of the response is plotted versus $\omega$ the forcing frequency, while in $b$ ) the pressure is shown for $\omega=0.6$-the initial conditions in b) coincide with the steady response for no fuel modulation. 
[12] HrRsh, C. Numerical Computation of Internal and External Flows, volume 2. Wiley-Interscience, New York, 1988.

[13] Darling, D., Radhakrishnan, K., and OyediRAN, A. Combustion noise at elevated pressures in a liquid-fueled premixed combustor. Technical Memorandum NASA TM-107481, ASME-97-GT308, NASA, June 1997. Turbo-Expo 97. 

Public reporting burden for this collection of information is estimated to average 1 hour per response, including the time for reviewing instructions, searching existing data sources, gathering and maintaining the data needed, and completing and reviewing the collection of intormation. Send somments regarding this burden estimate or any other aspect of this collection of information, including suggestions for reducing this burden, to Washington Headquarters Services. Directorate for Information Operations and Reports, 1215 Jefferson Davis Highway. Suite 1204, Arlington, VA 22202-4302, and to the Office of Management and Budget, Paperwonk Reduction Project (0704-0188), Washington. DC 20503.

\begin{tabular}{l|l|l|}
\hline 1. AGENCY USE ONLY (Leave blank) & 2. REPORT DATE & 3. REPOAT TYPE AND DATES COVERED
\end{tabular}

\begin{tabular}{|l|l|l} 
& November 1998 & Technical Memorandum \\
\hline
\end{tabular}

\section{TITLE AND SUBTITLE}

A Simplified Model for the Investigation of Acoustically

Driven Combustion Instabilities

6. AUTHOR(S)

D. Dane Quinn and Daniel E. Paxson
5. FUNDING NUMBERS

WU-523-26-13-00

8. PERFOAMING ORGANIZATION REPORT NUMBER

E-11267

National Aeronautics and Space Administration

Lewis Research Center

Cleveland, Ohio 44135-3191

10. SPONSORING/MONITORING AGENCY REPORT NUMBER

National Aeronautics and Space Administration

Washington, DC 20546-0001

NASA TM-1998-208488

AIAA-98-3764

\section{SUPPLEMENTARY NOTES}

Prepared for the 34th Joint Propulsion Conference cosponsored by AIAA, ASME, SAE, and ASEE, Cleveland, Ohio, July 12-15, 1998. D. Dane Quinn, The University of Akron, Department of Mechanical Engineering, Akron, Ohio 44325-3903 and Summer Faculty Fellow at NASA Lewis Research Cen er; Daniel E. Paxson, NASA Lewis Research Center. Responsible person, Daniel E. Paxson, organization code 5530, (216) 433-8334.

12a. DISTAIBUTION/AVAILABILITY STATEMENT

12b. DISTRIBUTION CODE

Unclassified - Unlimited

Subject Category: 07

Distribution: Nons:andard

This publication is available from the NASA Center for AeroSpace Information, (301) 621-0390.

13. ABSTRACT (Maximum 200 words)

A simplified one-dimensional model of reactive flow is presented which captures features of aeropropulsion systems, including acoustically driven combustion instabilities. Although the resulting partial differential equations are one dimensional, they qualitatively describe observed phenomena, including resonant frequencies and the admission of both steady and unsteady behavior. A number of simulations are shown which exhibit both steady and unsteady behavior, including flame migration and thermo acoustic instabilities. Finally, we present examples of unsteady flow resulting from fuel modulation.

14. SUBJECT TERMS

\begin{tabular}{|c|c|}
\hline \multirow{2}{*}{$\begin{array}{c}\text { 19. SECURIT } \\
\text { OF ABSTRACT } \\
\text { Unclassified }\end{array}$} & $\begin{array}{c}\text { 15. NUMBER OF PAGES } \\
16\end{array}$ \\
\cline { 2 - 2 } & $\begin{array}{c}\text { 16. PRICE CODE } \\
\text { AO3 }\end{array}$ \\
\hline & $\begin{array}{l}\text { Standard Form 298 (Rev. 2-89) } \\
\text { Prescribed by ANSI Std. Z39-18 } \\
298-102\end{array}$ \\
\hline
\end{tabular}

